\title{
Application of Light Scattering from Dielectric Cylinder Based upon Mie and Rayleigh-Gans-Born Theories to Polymer Systems. IV. An Application to Craze Formation in Glassy Polymers
}

\author{
Yukikazu Uemura, ${ }^{*}$ Kazunori TsujIEI, ${ }^{* *}$ Takeji Hashimoto, \\ and Hiromichi KAWAI \\ Department of Polymer Chemistry, Faculty of Engineering, \\ Kyoto University, Kyoto 606, Japan.
}

(Received July 17, 1979)

\begin{abstract}
The structure of the crazes developed in high-impact polystyrene films was studied by means of light scattering technique. The scattering intensity distributions which were measured both photometrically and photographically as a function of strain were analyzed in terms of scattering theories of Mie or Rayleigh-Gans-Born for an isolated and infinitely long cylinder with a size distribution as given by the zeroth-order logarithmic distribution function. The best fit between the measured and calculated scattering curves under $V_{\mathrm{V}}$ and $H_{\mathrm{H}}$ polarization conditions was accomplished by a simplex method, a method for the minimization of a mathematical function of several variables, the results of which yielded structure parameters characterizing the crazes in the glassy polymers, i.e., the refractive indices parallel and perpendicular to the stretching direction, the size distribution, and number of the craze centers.
\end{abstract}

KEY WORDS Light Scattering / Mie Theory / Rayleigh-Gans-Born Scattering / Crazing / Size Distribution / Glassy Polymers / High-Impact Polystyrene /

It is well known that an application of tensile stress to glassy polymers results in formation of crazes. ${ }^{1}$ The craze formation is associated with a localized yielding process and the first stage of fracture. The craze consists of polymer fibrils of 10 to $40 \mathrm{~nm}$ in diameter, interspersed by voids of 10 to $20 \mathrm{~nm}$ in diameter. The fibrils are a bundle of molecules oriented parallel to the stress direction. The boundaries between crazes and bulk polymer are sharp and oriented perpendicular to the stress direction. The thickness of the crazes perpendicular to the boundaries as observed by electron microscopy is typically of $0.1 \mu \mathrm{m}$ to $1.0 \mu \mathrm{m}$. The lateral dimensions of a craze are usually much larger than the thickness.

The structure of crazes has been studied most extensively by the transmission electron microscopy on ultrathin sections. A number of different tech-

* Present Address: Sumitomo Chemical Co., Ltd., 1-98, Naka 3-chome, Kasugade, Konohanaku, Osaka 554, Japan.

** Present Address: Mitsui Toatsu Chemicals Inc., Osaka Factory, Kamo, Takaishi-shi, Osaka 592, Japan. niques have been developed to prepare the ultrathin specimens for microscopy. ${ }^{1-5}$ Though the electron microscopic technique mentioned above has yielded the most direct evidence concerning the structure of crazes, it has the disadvantage that certain structural changes result in the process of preparing the specimens. Thus for quantitative studies of the structure of crazes it would be highly desirable to employ simultaneously some complementary techniques.

Kambour evaluated the refractive index of crazes and thus the density in the craze by measurement of the critical angle of incidence for the toral reflection of light at the craze boundary. ${ }^{6.7}$ In some cases, crazes show interference fringes, under vertical illumination, owing to reflection of light from their upper and lower boundaries. ${ }^{8}$ One can estimate the thickness of the crazes from the order of the fringes, if the refractive index is known. The small angle Xray scattering is also a useful technique to characterize the thickness of the craze parallel to the stress direction and diameter of the fibrils. However, the 
applications of the technique are still very limited at present stage. ${ }^{1 \mathrm{a}, 9,10}$

In this work we shall study laser-light scattering from crazed high-impact polystyrene films as one of the complementary techniques for characterizing the structure of crazes. We measure the $V_{\mathrm{V}}$ and $H_{\mathrm{H}}$ light scattering intensity distributions and fit the results with those calculated on the basis of the Mie theory for an isolated and infinitely long cylinder with a size distribution given by the zeroth order logarithmic function to evaluate the refractive indices of the crazes parallel and perpendicular to the stress direction, the average size of the craze (i.e., the diameter of the cylinder) in a direction perpendicular to the boundaries between the craze and bulk polymer, width of the size distribution, and number of crazes per unit volume.

\section{TEST SPECIMENS}

Four types of high-impact polystyrene (HIPS) commercially available from certain Japanese companies were used as test specimens. The HIPS is a graft copolymer of polystyrene onto a polybutadiene backbone and consists of a large fraction of polystyrene homopolymer and some homopolybutadiene as well. The specimens contain 5 to 8 $\mathrm{wt}^{\mathrm{o}} \%$ polybutadiene. The weight-average molecular weights of the polystyrenes are from $1.96 \times 10^{5}$ to $2.20 \times 10^{5}$.

The film specimens were prepared by a laboratory hot press. The pellets of HIPS were pressed at $190^{\circ} \mathrm{C}$ and quenched into an ice-water bath. The film specimens of about $80 \mu \mathrm{m}$ thick were cut into a

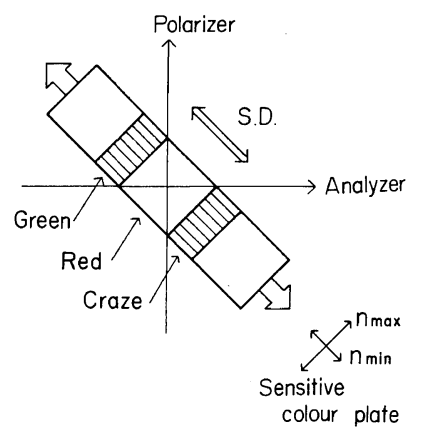

Figure 1. Schematic diagram showing retardation color of the stretched HIPS films under polarizing light microscope with the sensitive color plate. S.D., stretching direction. ribbon shape of $6 \times 35 \mathrm{~mm}^{2}$. The refractive index of the specimens was measured to be 1.60 .

Figure 1 represents a schematic diagram of photomicrographs of stretched HIPS films observed under polarizing light microscope with a sensitive color plate, the color plate being inserted with one of the principal optical axis corresponding to the fast component (i.e., small refractive index $n_{\min }$ ) parallel to the stretching direction (SD). The micrographs show localized, highly deformed regions and undeformed regions with their boundaries perpendicular to the stretching direction.

The undeformed region exhibits red retardation color, indicating an isotropic optical property, while the deformed region exhibits green color, typical of an optically anisotropic medium. The green color indicates that the refractive index along the SD is smaller than that perpendicular to the SD in the crazes. This manifests that the polystyrene molecules are aligned parallel to the SD in the deformed region, since polystyrene molecules have a negative birefringence, ${ }^{20}$ i.e., refractive index along the chain being smaller than that perpendicular to it. Thus the deformed region as it appeared in the green retardation color under the microscope should correspond to a craze or an assembly of crazes.

Figure 2 represents a typical electron micrograph showing the texture of the stetched HIPS films (SD in vertical). The specimens (\#1) were kept stretched by about $5 \%$ for a few days at room temperature, then released from the stretching device, stained in an aqueous solution of osmium tetroxide at room temperature. The specimens stained by osmium

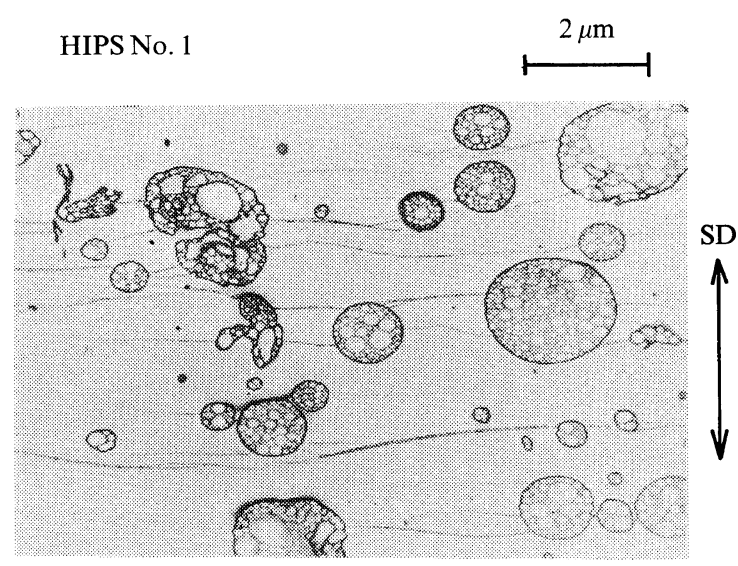

Figure 2. Typical transmission electron micrograph of ultra-thin section of the HIPS (specimen \#1) stained by osmium tetroxide. The stretching direction is vertical. 
tetroxide were cut in a plane parallel to the SD and perpendicular to the film surface into ultrathin sections with an ultramicrotome for electronmicroscopic observations. The micrograph clearly reveals a salami type texture typical of HIPS; the dark, stained regions of polybutadiene are dispersed in the unstained, light matrix of polystyrene. The size of the salami, i.e., the rubber particles which containd a high portion of polystyrene phase inside, has a wide distribution. The average-size of the particle may be described in terms of a spatial correlation distance, " $a$ ", a statistical parameter as obtained by Debye's plot of the scattering intensity distribution $^{11.12}$ as will be discussed later. The other HIPS specimens exhibited also a similar texture, their correlation distances being summarized in Table I.

Table I. Correlation distance " $a$ " (nm) of HIPS specimens

\begin{tabular}{ccc}
\hline \multicolumn{3}{c}{$a / \mathrm{nm}$} \\
\hline$\# 1$ & $\# 2$ & $\# 3$ \\
\hline 226 & 202 & 220 \\
\hline
\end{tabular}

The micrograph also clearly shows formation of crazes extended in a direction perpendicular to the stress direction. The observed size of the craze parallel to the SD, however, is expected to be much smaller than that under the stretched state due to the relaxation effect, the effect of which will also be discussed later.

Figure 3 shows optical micrographs of HIPS films (HIPS \#2) kept at constant elongations (0.3, 0.7, 2.0, and $3.3 \%$ ). Craze structures up to several microns in size are observed. The width, length, and number of crazes increases with elongation of the films. These tendencies are confirmed quantitatively as discussed later.

\section{LIGHT SCATTERING EXPERIMENTS}

Figure 4 represents typical laser light scattering patterns for the unstretched and stretched HIPS films (\#1) under $V_{\mathrm{V}}$ and $H_{\mathrm{H}}$ polarization conditions. The polarization directions of polarizer and analyzer are both vertical for $V_{\mathrm{V}}$ and horizontal for $H_{\mathrm{H}}$ polarization. the stretching direction is horizontal for both $V_{\mathrm{V}}$ abd $H_{\mathrm{H}}$ scatterings.

The $V_{\mathrm{V}}$ and $H_{\mathrm{H}}$ scattering patterns are almost

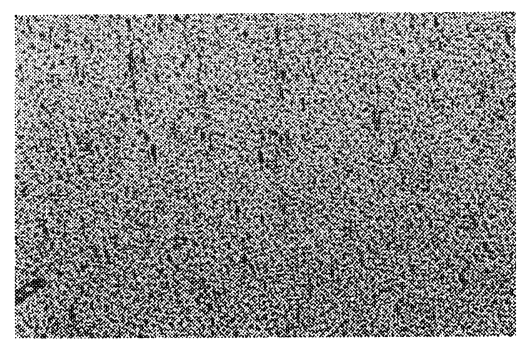

S.D.



$0.3(\%)$

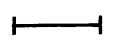

$100 \mu \mathrm{m}$

0.7

Figure 3. Optical micrographs of HIPS $\# 2$ kept at constant elongations.

identical, indicating that the spatial density fluctuations (i.e., the fluctuation in refractive index) is a principal contribution to the light scattering. The scattering patterns for the unstretched films are circularly symmetric with respect to the incident beam axis. Thus the density fluctuation is isotropic, reflecting the salami texture of the HIPS. The refractive index difference between the dispersed rubber phase and polystyrene matrix is small enough for the scattering to be capable of being described by Rayleigh-Gans-Born (R.G.B.) or Rayleigh-Gans 
HIPS No. 1
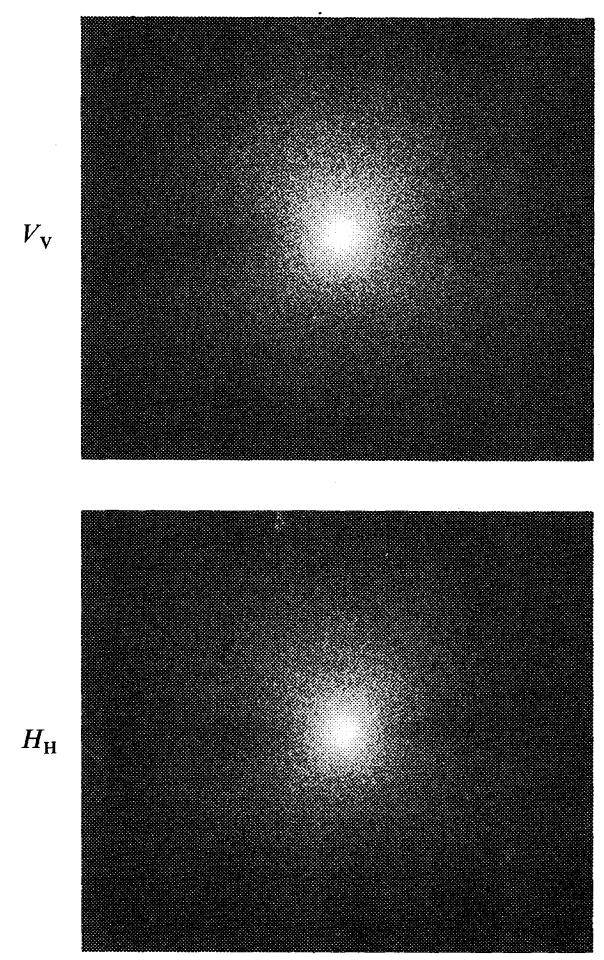

$0 \%$
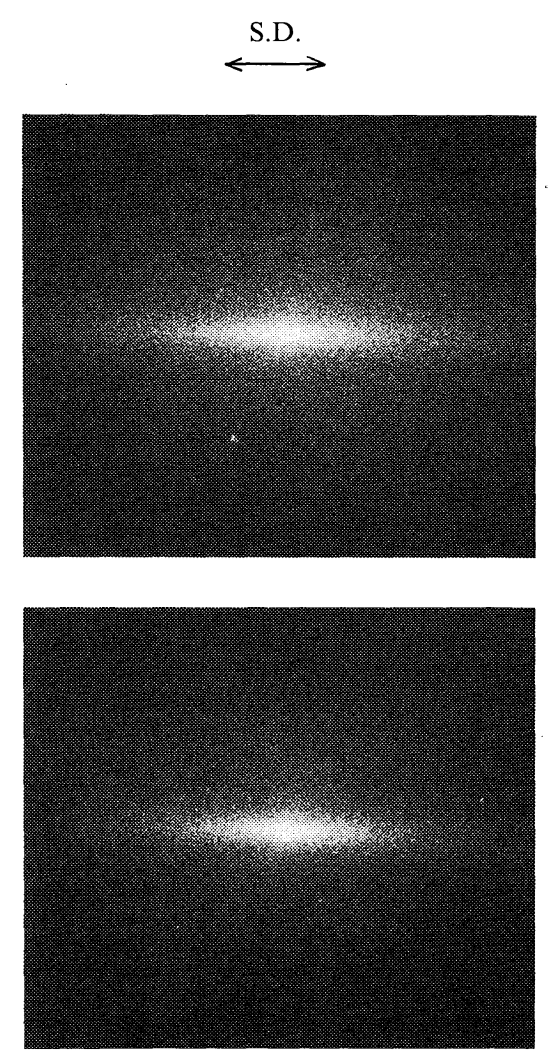

$0.9 \%$
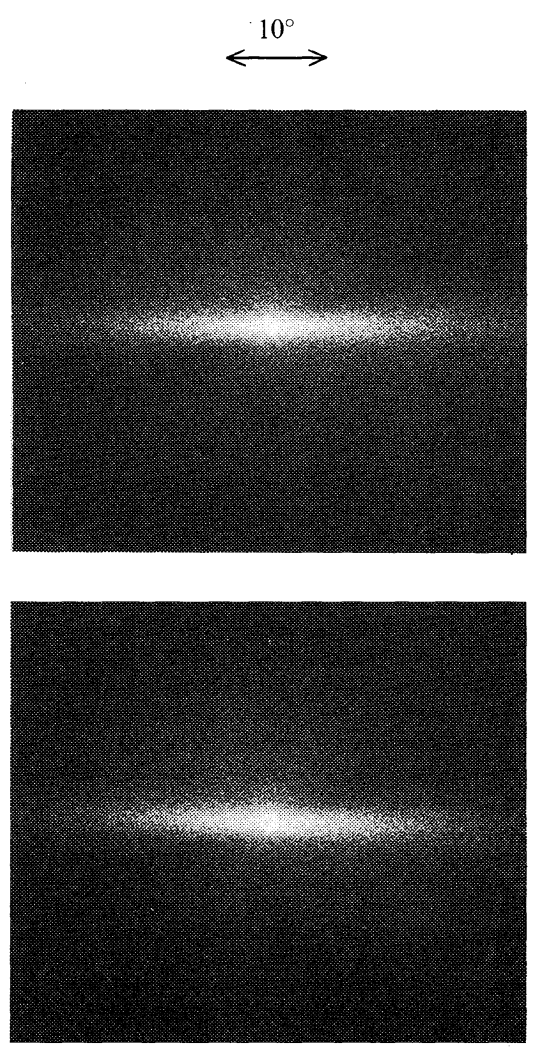

$2.7 \%$

Figure 4. Typical light scattering patterns as a function of strain percents for the specimen \#1. The stretching direction (S.D.) is horizontal. Both the polarizer and analyzer are set in vertical for $V_{\mathrm{V}}$ and in horizontal for $H_{\mathrm{H}}$ scattering. 
-Debye (R.G.D.) scattering (the scattering being described in the context of Born approximation). ${ }^{13,14}$

On stretching the specimens, very strong streaklike scattering appeared parallel to the SD. The scattering intensity was, much stronger than for the unstretched materials, indicating that new density heterogeneities are developed in a direction perpendicular to the stress direction as a consequence of the craze formation.

The change of the scattering intensity distribution along the horizontal direction of Figure 4 was quantitatively studied by scanning a photomultiplier in the horizontal plane. A He-Ne gas laser of $15 \mathrm{~mW}$ power was used as a light source. The polarization direction of the incident beam was varied by using a polarization rotator for the measurements of the $V_{\mathrm{V}}$ and $H_{\mathrm{H}}$ scattering. The excess scattering $\Delta I$ due to the craze formation was estimated by subtracting the scattered intensity for the unstretched films from that for the stretched films. The greater the difference in the scattering intensities between the unstretched and stretched films, the more accurate is the background subtraction procedure.

The excess scattering under the $V_{\mathrm{V}}$ and $H_{\mathrm{H}}$ polarizations was measured as a function of strain and scattering angles. Typical results are shown in Figure 5 for the specimen \#1 (open and solid circles) for $1.4 \%$ (curve A), $2.7 \%$ (curve B), and $3.3 \%$ (curve C) stretched specimens. The figure also contains the calculated scattering curves (solid line) which will be discussed later.

It should be noted that the scattering angle $\theta$ here and hereafter refers to the scattering angle in the medium but not the scattering angle in the air $\left(\theta_{\mathrm{a}}\right)$. The angle $\theta$ was estimated from the angle $\theta_{\mathrm{a}}$ with a knowledge of average refractive index of the scattering medium $\left(m_{\mathrm{s}}\right)$;

$$
\theta_{\mathrm{a}}=\arcsin \left(m_{\mathrm{s}} \sin \theta\right)
$$

It is clear that the excess scattering increases with increasing stretching and that the angular dissymmetry tends to decrease with increase stretching. These changes in the scattering curves are due to the changres of number of crazes per unit volume, size and distribution of size, and refractive index differences between the crazes and matrix. The quantitative analysis of the scattering curves will be discussed in the following sections. It may be worthwhile to point out that the intensity levels primarily depend on the number of crazes per unit volume.

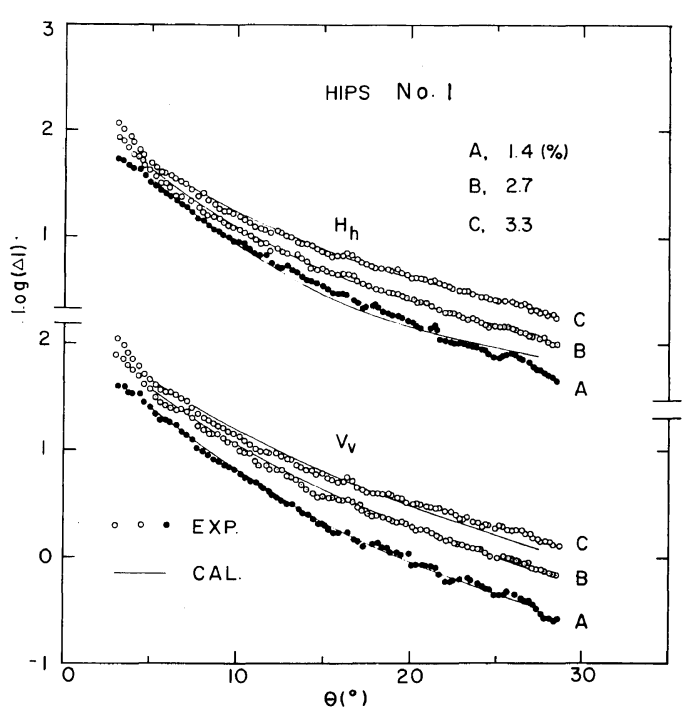

Figure 5. The excess scattering from the crazes as a function of the polarization conditions, scattering angles, and stretching percents for the specimen \#1. The solid lines are the calculated Mie scattering curves which are fitted with the measured curves by the simplex method.

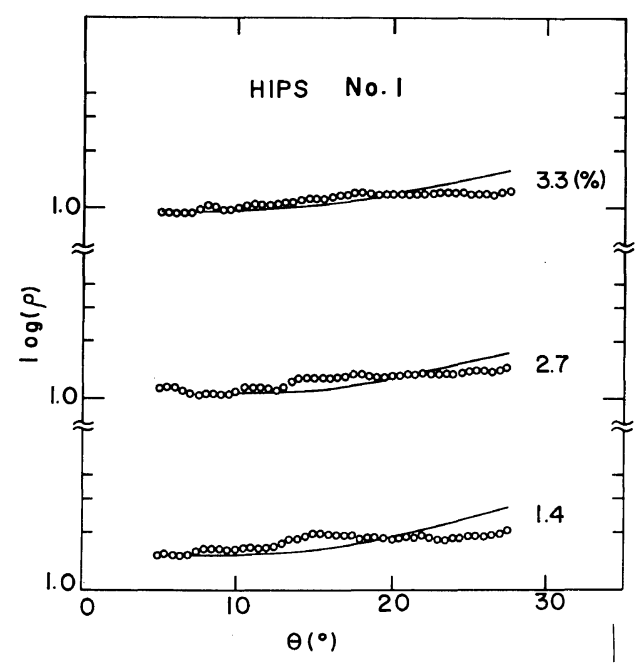

Figure 6. The intensity ratios of the $H_{\mathrm{H}}$ and $V_{\mathrm{V}}$ scattering from the crazes; $\rho(\theta)=\Delta I_{H_{\mathrm{h}}} / \Delta I_{V_{\mathrm{v}}}$. The open circles and the solid lines are the measured and calculated ratios, respectively. The calculated curves were obtained by using the same structure parameters as those for the curves in Figure 5. 


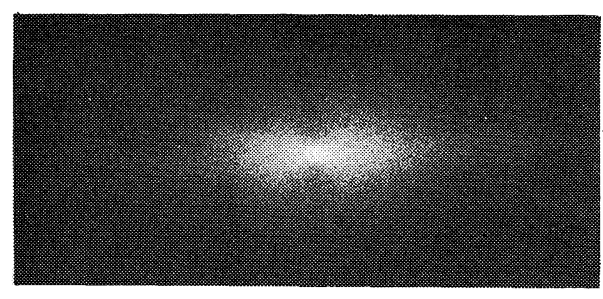

(a) $0 \mathrm{~min}$

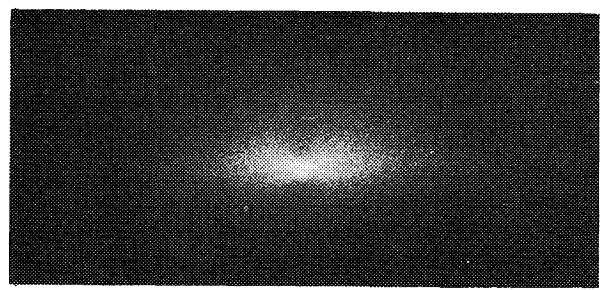

(b) $5 \mathrm{~min}$

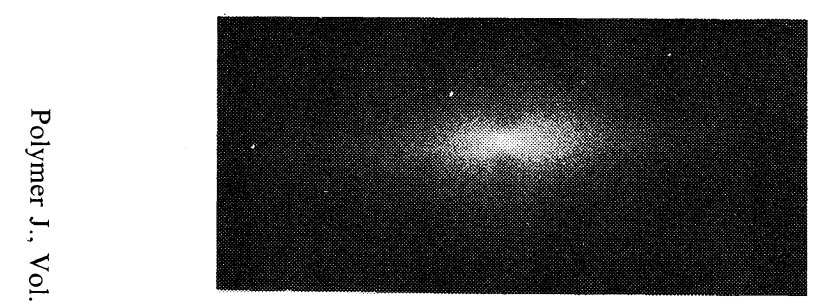

(c) $30 \mathrm{~min}$

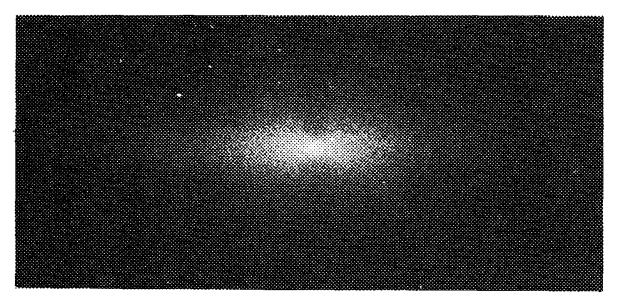

(d) $60 \mathrm{~min}$

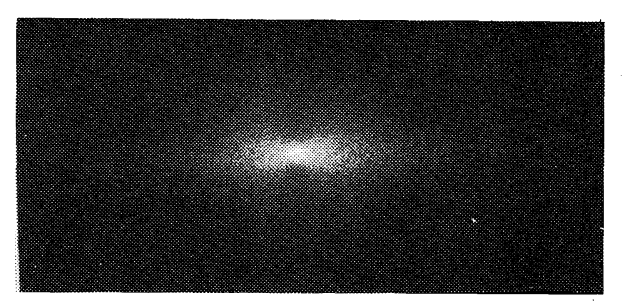

(e) $120 \mathrm{~min}$

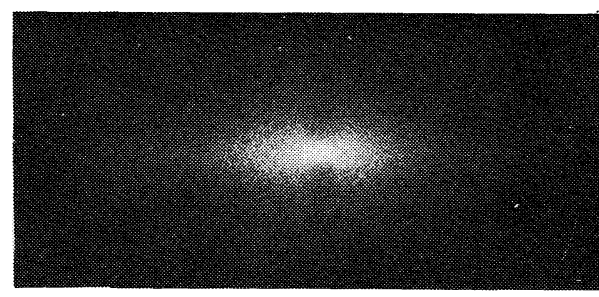

(f) $180 \mathrm{~min}$
HIPS No. 2

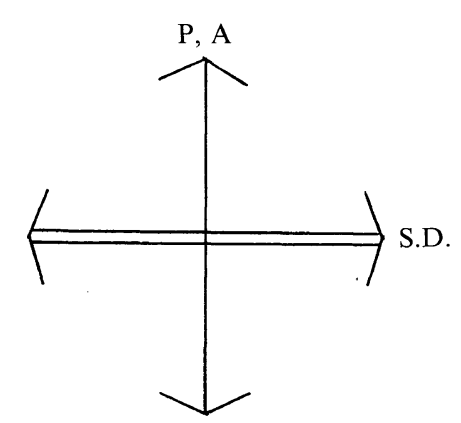

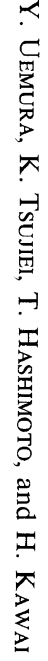



Figure 7. Change of $V_{\mathrm{v}}$ light scattering patterns as a function of time after a rapid stretching of the specimens $\# 2$ by about $2 \%$. 
Figure 6 shows the ratio of $H_{\mathrm{H}}$ to $V_{\mathrm{V}}$ scattering due to the craze as a function of stretching and scattering angles for the specimen $\# 1$.

$$
\rho(\theta)=\Delta I_{\mathrm{Hh}}(\theta) / \Delta I_{V_{\mathrm{v}}}(\theta)
$$

The figure also includes the calculated results (solid lines) which will be discussed later.

Similar data on $\Delta I_{V_{\mathrm{v}}}(\theta), \Delta I_{H_{\mathrm{h}}}(\theta)$ and $\rho(\theta)$ and similar changes of the scattering patterns with stretching ratio were obtained for the other specimens but they are not shown here.

Figure 7 shows experimental results on timeresolved light scattering experiments. The $V_{\mathrm{v}}$ scattering patterns were taken as a function of the time having elapsed after a rápid stretching of the specimen \#2 by about $2 \%$. It is seen from the figure that the shapes of the scattering patterns themselves do not change but the intensity level tends to increase with time.

The change of the intensity level was photometrically measured at two different scattering angles $\theta=12.3^{\circ}$ and $28.6^{\circ}$ in equatorial direction as in Figure 7 under $V_{\mathrm{v}}$ polarization as a function of the time after a rapid stretching of the specimens by $2 \%$ (left-half of each figure) or after a rapid removal of the stress (right-half of each figure) (Figure 8). The points a to $\mathrm{e}$ in the figure correspond to the times at which the scattering patterns in Figure 7 were taken. The intensity plotted here is not the excess scattering due to the craze but the total intensity, i.e., the excess scattering plus the scattering from the salami texture.

It is clear from the figure that the craze initiation and growth are quite slow at room temperature. A large amount of the intensity increases after stretching. The craze thus formed will then relax with time, resulting in a decrease in the scattering intensity as shown in the figure. In general one should be cautious in healing effect of the craze when interpreting the structure observed under an electron microscope.

Figure 9 shows an example of the change of the excess scattering intensity distributions under $V_{\mathrm{V}}$ and $H_{\mathrm{H}}$ polarizations at room temperature for the HIPS \#4 specimens. The curve A was obtained for the specimens in the stretched state $(2 \%)$ while the curves $\mathrm{B}$ and $\mathrm{C}$ were measured, respectively, $20 \mathrm{~h}$ and $44 \mathrm{~h}$ after the stress was removed. The change in the scattering curves suggests the healing effect of the craze structure such as average size, size distribution and density (or refractive indices) within the craze.
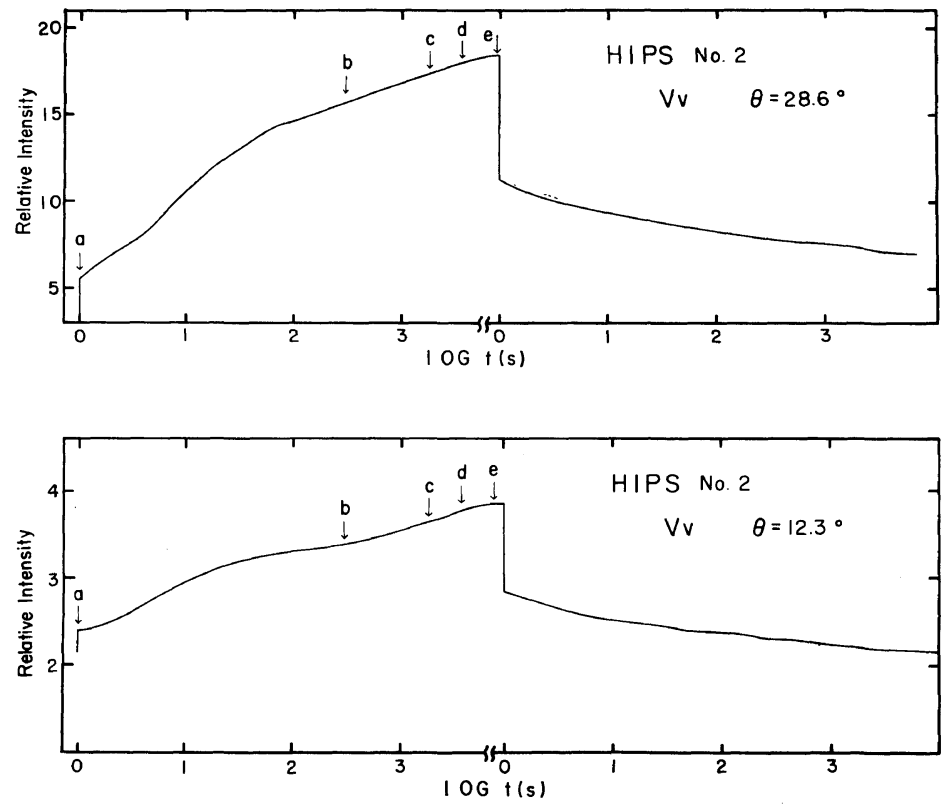

Figure 8. The change of the $V_{\mathrm{V}}$ scattering intensity at $\theta=12.3^{\circ}$ and $28.6^{\circ}$ as a function of time after a rapid stretching of the specimen \#2 by $2 \%$ (left-half of each figure) or following rapid removal of the stress (righthalf). The points a to e correspond to the instances at which the patterns in Figure 7 were taken. 


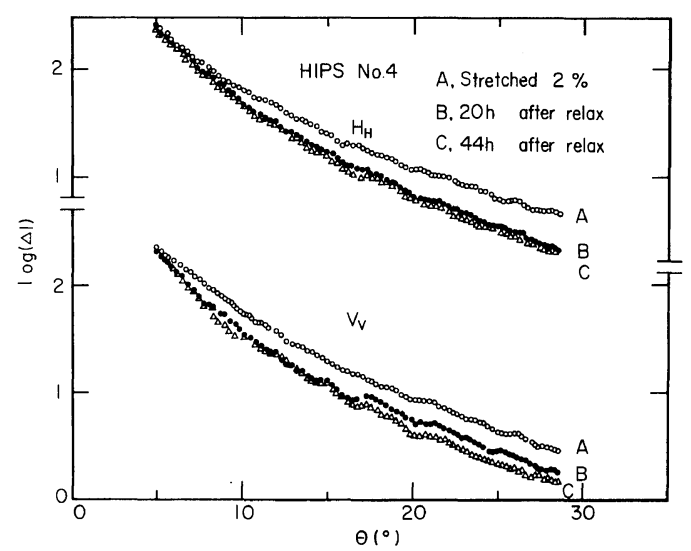

Figure 9. Change of excess scattering intensity distributions under $V_{\mathrm{V}}$ and $H_{\mathrm{H}}$ polarizations before and after removal of the stress at room temperature for the specimen \#4: curve A, stretched state; curve B, $20 \mathrm{~h}$; curve $\mathrm{C}, 44 \mathrm{~h}$ after removal of stress.

\section{ANALYSIS OF SCATTERING DATA}

Charactrization of Structure Under Unstretched State

The size of the salami texture typical of HIPS was characterized in terms of a statistical parameter, the so-called "correlation distance $a$ " of density (or refractive index) fluctuations. Since the difference between the refractive indices of the dispersed rubber-phase and of polystyrene matrix is relatively small and the density fluctuations is isotropic for the unstretched specimens, the scattering can be described by a Fourier transform of a spherical symmetric density correlation function $\gamma(r)$ in the context of the R.G.B. or R.G.D. approximation, ${ }^{11}$

$$
\begin{aligned}
& R_{V_{\mathrm{v}}}(h)=\frac{16 \pi^{4}\left\langle\eta^{2}\right\rangle}{\lambda_{0}^{4}} \int_{0}^{\infty} \mathrm{d} r \gamma(r) \frac{\sin h r}{h r} 4 \pi r^{2} \\
& R_{H_{\mathrm{h}}}(h)=\frac{16 \pi^{4}\left\langle\eta^{2}\right\rangle}{\lambda_{0}{ }^{4}} \cos ^{2} \theta \int_{0}^{\infty} \mathrm{d} r \gamma(r) \frac{\sin h r}{h r} 4 \pi r^{2}
\end{aligned}
$$

where $R(h)$ is the Rayleigh ratio defined as

$$
R(h)=I(\mathrm{~h}) D_{\mathrm{s}}{ }^{2} /\left(V I_{0}\right)
$$

$D_{\mathrm{s}}$ is the distance from the scattering materials to the detector, $V$ is the irradiated volume, $I_{0}$ is the incident beam intensity, and $\lambda_{0}$ is the wavelength in vacuo. The quantity $h$ is a reduced scattering angle defined as

$$
h=(4 \pi / \lambda) \sin (\theta / 2)
$$

where $\lambda$ is the wavelength of light in the medium. The quantity $\eta$ is the fluctuation about the average value $\alpha_{0}$ of polarizability $\alpha$ of the scattering element at $r$,

$$
\eta(\boldsymbol{r})=\alpha(\boldsymbol{r})-\alpha_{0}
$$

and the correlation function is defined by

$$
\gamma(r)=\left\langle\eta\left(\boldsymbol{r}_{\mathrm{i}}\right) \eta\left(\boldsymbol{r}_{\mathrm{j}}\right)\right\rangle_{\boldsymbol{r}} /\left\langle\eta^{2}\right\rangle
$$

where $r \equiv\left|r_{\mathrm{i}}-\boldsymbol{r}_{\mathrm{j}}\right|$.

Debye-Anderson-Brumberger ${ }^{12}$ found that the correlation function is given by

$$
\gamma(r)=\exp (-r / a)
$$

where $a$ is the so-called correlation distance. It follows then from eq $3-5$ and 9 ,

$$
I_{V_{\mathrm{v}}}(h)=(\text { const })\left(1+a^{2} h^{2}\right)^{-2}
$$

Thus, the correlation distance is obtained from

$$
a=\left[\frac{\left(\text { slope of the plot } I_{V_{\mathrm{v}}}^{-1 / 2} v s . h^{2}\right)}{\text { (intercept of the } I_{V_{\mathrm{v}}}^{-1 / 2} \text { at } h^{2}=0}\right]^{1 / 2}
$$

\section{Characterization of the Structure of Craze}

Model. In this work, we analyzed the structure of crazes by analyzing the excess scattering due to crazes for normal incidence under $V_{\mathrm{V}}$ and $H_{\mathrm{H}}$ polarizations. Figure 10 defines the $V_{\mathrm{V}}$ and $H_{\mathrm{H}}$ polarization conditions, model for a craze, and



Figure 10. The model of infinitely long cylinder for the craze, and the relation between the craze, stretching direction, the film surface, propagation direction of the incident beam (unit vector $s_{0}$ ), scanning plane, and polarization directions of the polarizer and analyzer. 
scanning plane. The single craze developed perpendicularly to the stretching direction was simplified by an isolated cylinder of infinite length. The stress direction is set parallel to $Y$-axis, and thus the lateral direction of the craze or the axis of the cylinder is set parallel to $Z$-axis. The incident beam propagates along $X$-axis (unit vector $s_{0}$ ) and we observe the scattering as a function $\theta$ in $X Y$ plane. The polarization directions of polarizer and analyzer are set parallel to $Z$-axis for $V_{\mathrm{v}}$ scattering, while they are set perpendicular to the $Z$-axis for $H_{\mathrm{H}}$ scattering as shown in the figure.

The craze or the cylinder has uniaxial symmetric optical anisotropy, having refractive indices $m_{1}$ and $m_{2}$ along $Z$ - and $Y$-axis, respectively, and is embedded in a matrix having an average refractive index of the specimen, $m_{\mathrm{s}}=1.60$. We assume that the centers of the crazes are distributed randomly along $Y$-axis, thus the inter-particle interference effect does not play an important role on the observed scattering. The scattering intensities from a single cyclinder, $I_{V_{\mathrm{v}}}\left(\theta ; R, m_{1}\right)$ and $I_{H_{1}}\left(\theta ; R, m_{2}\right)$, are given in terms of R.G.B. scattering or Mie scattering theories.

We assume that the size distribution of the crazes is given by the zeroth-order logarithmic distribution (ZOLD) function,

$$
\begin{aligned}
& P(R)= \\
& \quad \exp \left(-\left(\ln R-\ln R_{\mathrm{M}}\right)^{2} / 2 \Delta^{2}\right) / \sqrt{2 \pi} \Delta R_{\mathrm{M}} \exp \left(\Delta^{2} / 2\right)
\end{aligned}
$$

This function is frequently used to describe the effect of the polydispersity in the scattering studies. ${ }^{14}$ The quantity $R_{\mathrm{M}}$ is a modal value and $\Delta$ is the parameter characterizing the width of the distribution (characterization of this function being shown in Figures 16 to 18 ).

The average scattering intensities under $V_{\mathrm{V}}$ and $H_{\mathrm{H}}$ polarizations are then given by

$$
\begin{aligned}
\Delta I_{V_{\mathrm{v}}}(\theta) & =\int_{0}^{\infty} \mathrm{d} R P(R) I_{V_{\mathrm{v}}}\left(\theta ; R, m_{1}\right) \\
\Delta I_{H_{\mathrm{h}}}(\theta) & =\int_{0}^{\infty} \mathrm{d} R P(R) I_{H_{\mathrm{h}}}\left(\theta ; R, m_{2}\right)
\end{aligned}
$$

Obviously the scattering intensity is a function of many variables; $R_{\mathrm{M}}, \Delta, m_{1}$ and $m_{2}$. We will find an optimum set of parameters by fitting the calculated curves to the experimentally measured scattering curves based on the simplex method. ${ }^{15}$ This method will be briefly described in the next section.

Simplex Method to Obtain An Optimum Set of Parameters. The simplexes method is one of the mathematical methods to minimize a function of $n$ variables. ${ }^{15}$ Let $\boldsymbol{X}$ represents a set of parameters to be evaluated. The functions $I\left(\theta_{i}, \boldsymbol{X}\right)$ and $I_{\exp }\left(\theta_{i}\right)$ are the theoretical and experimental values, respectively, at each scattering angle $\theta_{i}$. Let $W(\mathbf{X})$ be defined as,

$$
W(\boldsymbol{X})=\sum_{i}\left[I\left(\theta_{i}, \boldsymbol{X}\right)-I_{\exp }\left(\theta_{i}\right)\right]^{2}
$$

In the simplex method, the function $W(\boldsymbol{X})$ is minimized by varying the set of parameters $\boldsymbol{X}$. The minimization is conducted by comparing the function values $W(\boldsymbol{X})$ at the $(n+1)$ vertices of a general simplex, followed by the replacement of the vertex with the highest value by another point. Thus the simplex adapts itself to the local landscape and contracts on the final minimum.

\section{Scattering Theories}

The basic scattering theories of Mie and of R.G.B. from a simple cylinder or coaxial cylinder are described in detail in previous papers of this series. ${ }^{16-18}$

The R.G.B. scattering from an infinitely long cylinder is given by

$$
I_{V_{\mathrm{v}}}\left(\theta ; R, m_{1}\right)=(\text { const })\left(m_{1}-m_{\mathrm{s}}\right)^{2}\left[J_{1}(U) / U\right]^{2}
$$

and

$$
I_{H_{\mathrm{h}}}\left(\theta ; R, m_{2}\right)=\text { (const) }\left(m_{2}-m_{\mathrm{s}}\right)^{2} \cos ^{2} \theta\left[J_{1}(U) / U\right]^{2-}
$$

where

$$
U=4 \pi(R / \lambda) \sin (\theta / 2)
$$

$J_{n}$ is the first kind Bessel function of the order $n$.

Mie scattering from the same cylinder is given by

$$
I_{V_{\mathrm{v}}}\left(\theta ; R, m_{1}\right)=\text { (const) }\left|b_{0}+2 \sum_{n=1}^{\infty} b_{n} \cos n \theta\right|^{2}
$$

where

$$
b_{n}=\frac{m_{1} J_{n}\left(m_{\mathrm{s}} \beta\right) J_{n}^{\prime}\left(m_{1} \beta\right)-m_{\mathrm{s}} J_{n}^{\prime}\left(m_{\mathrm{s}} \beta\right) J_{n}\left(m_{1} \beta\right)}{m_{1} H_{n}\left(m_{\mathrm{s}} \beta\right) J_{n}^{\prime}\left(m_{1} \beta\right)-m_{\mathrm{s}} H_{n}^{\prime}\left(m_{\mathrm{s}} \beta\right) J_{n}\left(m_{1} \beta\right)}
$$

$H_{n}$ is the second kind Hankel function of the order $n$ and 


$$
\begin{aligned}
& J_{n}^{\prime}\left(m_{1} \beta\right)=\left(\mathrm{d} J_{n}(x) / \mathrm{d} x\right)_{x=m_{1} \beta} \\
& H_{n}^{\prime}\left(m_{\mathrm{s}} \beta\right)=\left(\mathrm{d} H_{n}(x) / \mathrm{d} x\right)_{x=m_{\mathrm{s}} \beta} \quad \text { and etc. }
\end{aligned}
$$

The quantity $\beta$ is defined as

$$
\beta=2 \pi\left(R / \lambda_{0}\right)
$$

Similarly $H_{\mathrm{H}}$ scattering is given by

$$
I_{H_{\mathbf{h}}}\left(\theta ; R, m_{2}\right)=\text { (const) }\left|a_{0}+2 \sum_{n=1}^{\infty} a_{n} \cos n \theta\right|^{2}
$$

where

$$
a_{n}=\frac{m_{\mathrm{s}} J_{n}\left(m_{\mathrm{s}} \beta\right) J_{n}^{\prime}\left(m_{2} \beta\right)-m_{2} J_{n}\left(m_{2} \beta\right) J_{n}^{\prime}\left(m_{\mathrm{s}} \beta\right)}{m_{\mathrm{s}} H_{n}\left(m_{\mathrm{s}} \beta\right) J_{n}^{\prime}\left(m_{2} \beta\right)-m_{2} H_{n}^{\prime}\left(m_{\mathrm{s}} \beta\right) J_{n}\left(m_{2} \beta\right)}
$$

The equations 16 and 17 or 19 and 23 are substituted in eq 13 and 14 to obtain the average scattering intensity from the craze. The calculated intensity is then fitted with the measured intensity by using the simplex method to obtain parameters characterizing the structure of craze.

\section{RESULTS}

\section{Effects of Various Parameters on the Scattering} Curves

It should be worthwhile to examine some effects of varying each parameter on the calculated Mie scattering curves. The results are shown in Figures $11-$ 13 , in which the relative scattered intensity under the $V_{\mathrm{v}}$ polarization condition $\left(I / I_{0}\right)$ are plotted as a function of the scattering angles for a given value of $m_{\mathrm{s}}=1.6$. For the sake of simplicity, we ignored the small the anisotropy in refractive indices, viz., we set $m_{1}=m_{2}=m$. The dependence of the light scattering on the refractive index $m$ of the cylinder is shown in Figure 11 for a set of parameters; $R_{\mathrm{M}}=1.0 \mu \mathrm{m}$, $\Delta=0.5$, and $m_{\mathrm{s}}=1.6$. With increasing values of $m$, the difference between the refractive indices of the scattering particle and the medium becomes smaller, and therefore the scattering from the system approaches the criterion of the R.G.B. scattering. In this limiting case, the intensity drops very rapidly as $\theta$ increases. With decreasing values of $m$, the intensity levels at the high scattering angles increase, leading to less angularly dependent scattering intensity distributions. This is characteristic of the Mie scattering compared to the R.G.B. scattering.

Figure 12 shows an effect of $R_{\mathrm{M}}$, the modal value of

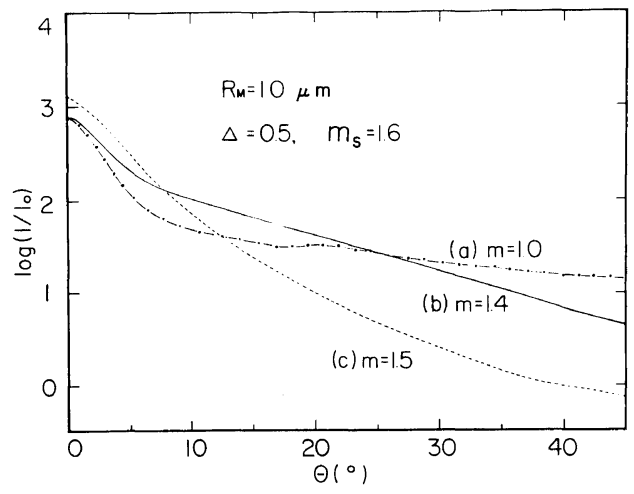

Figure 11. The calculated Mie scattering intensity for various values of $m=m_{1}=m_{2}$ for a given set of the parameters, $R_{\mathrm{M}}=1.0 \mu \mathrm{m}, \Delta=0.5$, and $m_{\mathrm{s}}=1.6$.

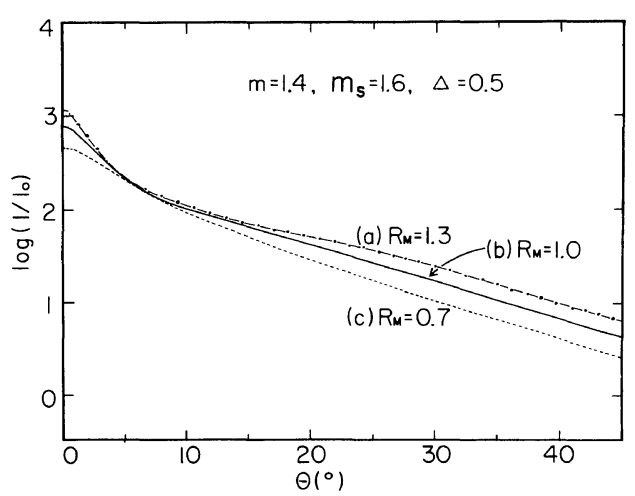

Figure 12. The calculated Mie scattering intensity for various values of $R_{\mathrm{M}}$ for a given set of the parameters, $m=1.4, m_{\mathrm{s}}=1.6$, and $\Delta=0.5$.



Figure 13. The calculated Mie scattering intensity for various values of $\Delta$ for a given set of the parameters, $R_{\mathrm{M}}=1.0 \mu \mathrm{m}, m=1.4$, and $m_{\mathrm{s}}=1.6$. 
Mie and Rayleigh-Gans-Born Scattering from Polymer Composite. IV.

the size distribution for a given set of the parameters; $m=1.4, m_{\mathrm{s}}=1.6$, and $\Delta=0.5$. By increasing $R_{\mathrm{M}}$, the intensity level at very small angles $\left(\theta<5^{\circ}\right)$ increases and the intensity tends to drop more rapidly with increasing $\theta$. This tendency seems to be reasonable since the greater the value of $R_{\mathrm{M}}$, the greater the contribution of scattering from large particles. At large scattering angles $\left(\theta>5^{\circ}\right)$, a system having a greater value of $R_{\mathrm{M}}$ has a higher intensity level and a less angularly dependent scattering profile, the tendency of which cannot be explained in terms of the R.G.B. scattering theory.

Figure 13 shows the effect of the width $\Delta$ of the size distribution for a given set of the parameters; $R_{\mathrm{M}}=1.0 \mu \mathrm{m}, m=1.4$, and $m_{\mathrm{s}}=1.6$. The increase of $\Delta$ may have qualitatively the same effect as an increase in the contribution from the large particles.

Close observation of the results in Figures 11-13 indicates that each parameter affects the scattering in a different manner, facilitating the assessment of the structure parameters by the best fittings of the experimental and calculated scattering curves.

\section{Remarks on the Guinier Plot}

Guinier derived the scattering at very small angles to be described, in the context of the R.G.B. approximation, in terms of a Gaussian form, ${ }^{19}$

$$
I(\boldsymbol{h})=I(\boldsymbol{h}=0) \exp \left(-h^{2} D^{2}\right)
$$

where $D$ is an average inertial distance of the particle with respect to the particular plane through the center of the particle perpendicular to the scattering vector $\boldsymbol{h}$. In our special circumstances, it follows that

$$
D=R / 2
$$

Equation 25 is sometimes used to estimate the size of the particles from a Guinier plot (i.e., the plot of $\ln I$ $v s . h^{2}$ ) beyond its theoretical limitation that the systems must satisfy the R.G.B. criterion. Therefore we estimate the error involved when the Guinier plot is applied beyond the theoretical limitation. Figure 14 shows the Guinier plot for a single cylinder having a radius of $1.0 \mu \mathrm{m}$ embedded in the matrix of $m_{\mathrm{s}}=1.6$ for various values of the refractive index $m$ of the cylinder. The R.G.B. criterion is rigorously satisfied for the cylinder having $m=1.599$. Consequently the plot yielded an accurate size of the cylinder. However, by decreasing the value $m$, the deviation from the R.G.B. approximation becomes greater, thus giving rise to an incorrect value for $R$ as shown

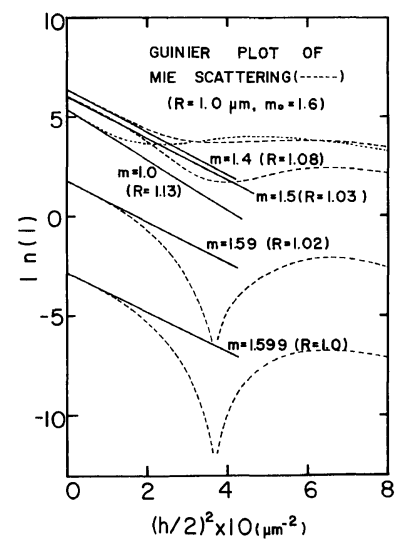

Figure 14. Guinier plots for a single cylinder having the radius $1.0 \mu \mathrm{m}$ and various values of refractive index $m\left(=m_{1}=m_{2}\right)$ embedded in the matrix of $m_{\mathrm{s}}=1.6$. The Mie effects are shown to give larger apparent size.

in the figure. The error, however, (which increases with increases of the size and of the difference in refractive indices) is surprisingly small (at most $13 \%$, in this special case), because the R.G.B. approximation is a good approximation for small angle scattering for which the Guinier plot is applied. Generally the Mie effect tends to give an estimation of size greater than it actually is.

\section{Correlation Distance for the Underformed Specimens}

Figure 15 shows a typical Debye plot for the HIPS \#1 specimens. Similar plots were obtained for the other specimens. The correlation distance, " $a$ " was evaluated from the plots by using eq 11 , the results of which are summarized in Table I. The results



Figure 15. Typical Debye-plot for the HIPS \#1 specimen. 
indicate that average sizes of the texture typical of the HIPS are essentially the same for all specimens.

Results of Curve-Fittings of the Measured and Calculated Scattering Curves by the Simplex Method

The measured relative intensity distributions of the excess scattering under $V_{\mathrm{V}}$ and $H_{\mathrm{H}}$ polarization conditions were fitted with the calculated curves by the simplex method: The intensity ratios of the $H_{\mathrm{H}}$ and $V_{\mathrm{V}}$ scattering, $\rho=\Delta I_{H_{\mathrm{h}}} / \Delta I_{V_{\mathrm{v}}}$ are also important and their values were also fitted as a function of the scattering angles and the strain. Figures 5 and 6 shows typical results obtained for the specimen $\# 1$ as a function of the strain. The measured curves (shown by the points) fit well with the calculated curves (solid lines) over a sufficiently wide scattering angles. Similar results were obtained for the other specimens although they will not be shown here.

The change in the craze size for these specimens is shown in Figure 16 as a function of strain $\varepsilon(\%)$. With increasing strain, the average size becomes larger and the size distribution becomes broader. The modal values of the craze radius are of the order of several hundred nanometers.

In Table II is shown the change of the refractive indices $m_{1}$ and $m_{2}$ as estimated from the curve fittings. It is shown that the refractive indices $m_{1}$ and $m_{2}$ are much less than $m_{\mathrm{s}}=1.6$ of the matrix but much greater than unity, indicating that the structure developed by a localized deformation is a craze but not a crack (the refractive index of a pure crack should be unity). Several runs of the simplex from



Figure 16. Estimated size distributions for the specimen \#1 stretched by $1.4 \%$ (curve A), $2.7 \%$ (curve B), and $3.3 \%$ (curve $(\mathrm{C})$.

different initial parameters gave the same final parameter values within $1 \%$ error for $m_{1}$ and $m_{2}, 10 \%$ for $R_{\mathrm{M}}$ and $20 \%$ for $\Delta$ values.

By assuming the additivity of refractive indices of the polymer $\left(m_{\mathrm{p}}=m_{\mathrm{s}}=1.6\right)$ and the air $\left(m_{\mathrm{a}}=1.0\right)$ in the crazed region, one may qualitatatively evaluate the volume fraction $\phi_{\mathrm{p}}$ of the polymer in the crazes.

$$
m_{1} \cong m_{2}=m_{\mathrm{p}} \phi_{\mathrm{p}}+m_{\mathrm{a}}\left(1-\phi_{\mathrm{p}}\right)=0.6 \phi_{\mathrm{p}}+1
$$

The volume fractions thus estimated from the values of $m_{1} \cong m_{2}$ are also included in the Table. With increasing strain, the refractive indices $m_{1}$ and $m_{2}$ tend to decrease, indicating that the volume fraction of polymer, $\phi_{\mathrm{p}}$, tends to decrease with increasing strain. The volume fractions estimated agree with the

Table II. The structure parameters of the crazes of HIPS specimens

\begin{tabular}{|c|c|c|c|c|c|c|c|}
\hline \multirow{2}{*}{$\begin{array}{l}\text { Specimen } \\
\text { code }\end{array}$} & \multirow{2}{*}{ Strain $/ \%$} & \multicolumn{2}{|c|}{ Refractive indices } & \multirow{2}{*}{$R_{\mathrm{M}} / \mu \mathrm{m}$} & \multirow{2}{*}{$\Delta$} & \multirow{2}{*}{$N$} & \multirow{2}{*}{$\phi_{\mathrm{p}} / \%$} \\
\hline & & $m_{1}$ & $m_{2}$ & & & & \\
\hline \multirow{3}{*}{$\# 1$} & 1.4 & 1.47 & 1.47 & 0.72 & 0.38 & 1 & 78 \\
\hline & 2.7 & 1.46 & 1.46 & 0.79 & 0.49 & 1.16 & 77 \\
\hline & 3.3 & 1.45 & 1.43 & 0.75 & 0.50 & 1.51 & 73 \\
\hline \multirow{3}{*}{$\# 2$} & 1.8 & 1.49 & 1.49 & 0.63 & 0.38 & 1 & 82 \\
\hline & 3.6 & 1.47 & 1.47 & 0.64 & 0.42 & 1.37 & 78 \\
\hline & 4.7 & 1.45 & 1.43 & 0.74 & 0.50 & 2.02 & 73 \\
\hline \multirow{3}{*}{$\# 3$} & 1.2 & 1.48 & 1.47 & 0.66 & 0.46 & 1 & 79 \\
\hline & 1.5 & 1.47 & 1.46 & 0.67 & 0.47 & 1.81 & 78 \\
\hline & 1.8 & 1.46 & 1.46 & 0.76 & 0.49 & 2.39 & 77 \\
\hline
\end{tabular}


Mie and Rayleigh-Gans-Born Scattering from Polymer Composite. IV.

values obtained by Kambour ${ }^{6.7}$ for some glassy polymers, though they are somewhat higher. One of the reasons for the discrepancies is the difference of the measuring methods. Moreover, the stretching percents in the present study are smaller than those in Kambour's experiments.

The $V_{\mathrm{V}}$ and $H_{\mathrm{H}}$ intensity levels tend to increase with increasing strain as a consequence of the increasing number of craze centers. The relative increase in the number of the crazes $(N)$ as determined from the intensity levels is also shown in the Table. For the specimen $\# 1, N$ is the number relative to the number of crazes developed at strain 1.4 percents.

The results for the other specimens are shown in Figure 17 and 18, and are summarized in the Table II.

It is generally concluded from the Table II that the deformed regions in all the specimens have refractive indices from 1.43 to 1.49 , suggesting the formation of crazes but not cracks. It is also shown that the refractive index $\left(m_{1}\right)$ perpendicular to the stress direction is slightly larger than that $\left(m_{2}\right)$ parallel to it, indicating that the polystyrene molecules in the crazes (or bundles of the molecules) orientate parallel to the stress direction. The refractive indices of crazes and thus the volume fraction of polymers $\phi_{\mathrm{p}}$ in the crazes tend to decrease with increasing strain as shown in Figure 19.

It is also concluded that the average size of the crazes and the distribution of the craze size increase with increasing strain as shown in Figure 20. There

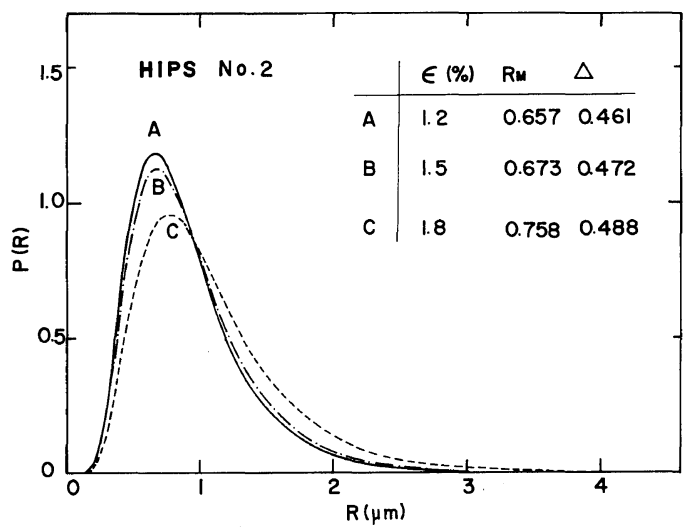

Figure 17. Estimated size distribution for the specimen \#2 stretched by $1.2 \%$ (curve A), $1.5 \%$ (curve B), and $1.8 \%$ (curve C).



Figure 18. Estimated size distribution for the specimen \#3 stretched by $1.8 \%$ (curve A), 3.6\% (curve B), and $4.7 \%$ (curve C).

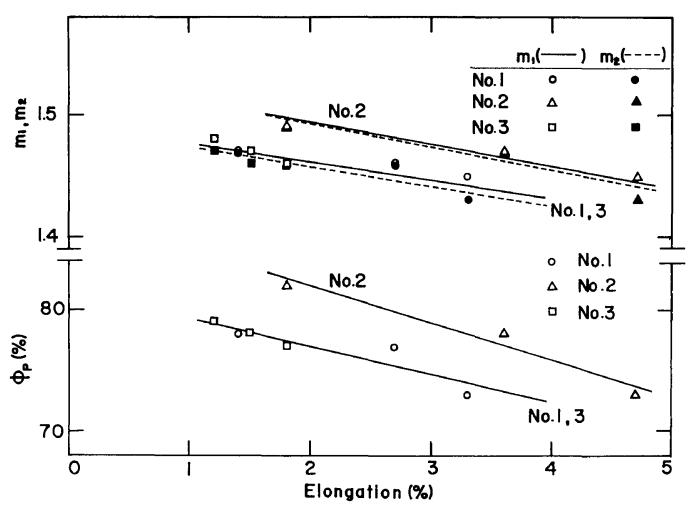

Figure 19. Estimated values for the refractive indices of the crazes $m_{1}$ and $m_{2}$, and for the volume fraction of polymer in the crazes, $\phi_{\mathrm{p}}$ as a function of strain.

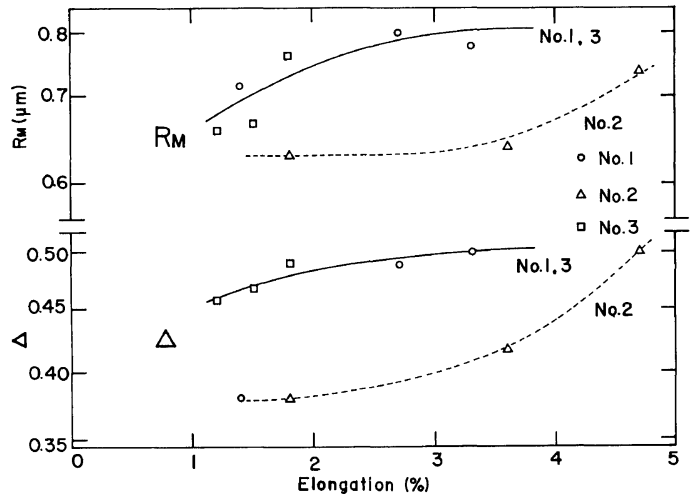

Figure 20. Estimated values for $R_{\mathrm{M}}$ and $\Delta$ as a function of strain. 
seems to be a distinct difference in the craze formation between the two groups of the specimens, i.e., the results for the specimens $\# 1$ and $\# 3$ are similar to each other but are different from those for the specimen \#2, although the underformed textures are almost identical, having the same spatial correlation distance.

It is beyond the scope of this paper to clarify the difference in terms of the differences in the chemistry of the HIPS such as molecular weight and its distribution for each component, fractional composition of polybutadiene, microstructure of polybutadiene and etc. Yet, this problem and its relation to the physical properties of the HIPS should be the most important problems to be solved in future.

The number of the crazed centers is shown to increase with increasing strain.

In this analysis we adopted as the model for the geometry of the craze an infinitely long cylinder with its cylindrical axis perfectly oriented to the stress direction. This cylindrical model is chosen simply to reduce the computing time as much as possible without loosing the physical significance about the scattering from the craze. The model of a thin lamella or thin disk of thickness $T$ with its boundary normal (unit vector $\boldsymbol{n}$ ) perfectly oriented parallel to the stress direction ( $Y$-axis) may be a better model for the craze (Figure 21). However, the scattering from such systems should not differ significantly from that of the cylindrical craze, provided that the incident beam is irradiated normal to the film surface, and thus normal to $\boldsymbol{n}$, as in our experiment. Under this

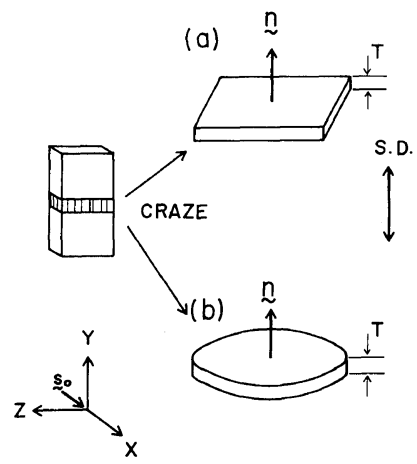

Figure 21. Lamellar (a) and disk-like model (b) for the craze; $\boldsymbol{n}$ and $\boldsymbol{s}_{0}$ are the unit vectors along the stretching direction and the propagation direction of the incident beam, respectively. $X Y$ plane is the scanning plane of the scattered light. circumstance, the scattering intensity distribution is not very sensitive to the dimensions of the structure perpendicular to $\boldsymbol{n}$ but depends primarily on the thickness $T$ or radius $R$ of the cylinder. Thus the size and its distribution evaluated on the basis of the cylinder model should have, at least, qualitative significances, although the estimated parameters quantitatively depend on particular models selected. The cylinder diameter is supposed to correspond to the thickness of the craze band or the lamella thickness $T$ in Figure 21.

The light scattering method estimates the contribution of the particles with large diameters more than the small ones. Moreover the scattering tends to count an aggregate of particles as a single particles, if the interparticle interference effects within the aggregate are strong. Such interference effects are generally significant at small angles and vanishes at large scattering angles.

In this regard we did not utilize the scattering data at small angles less than $\theta=5^{\circ}$. It may be worth noting that the curve fittings of the data in the angular region greater than $10^{\circ}$ gives a slightly smaller $R_{\mathrm{M}}$ values (typically several percent smaller) than those in the angular region greater than $5^{\circ}$. This is due to the neglect of the contribution of large particle diameters or due to the fact that the interparticle interference effect is still significant in the

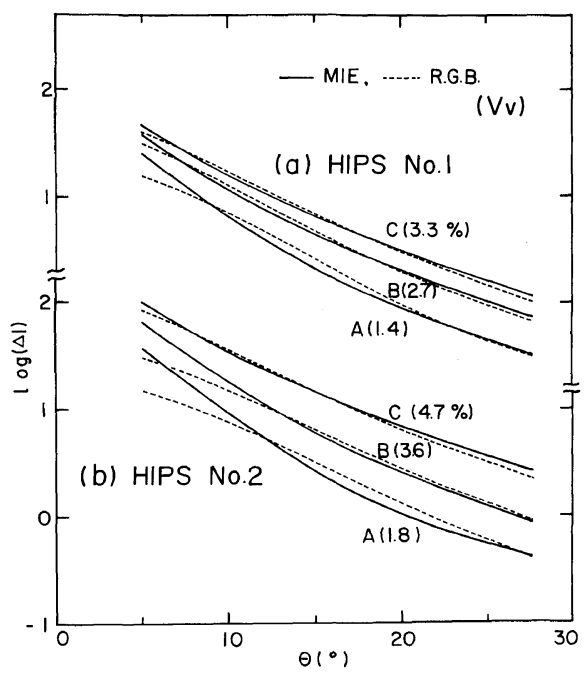

Figure 22. The calculated Mie (solid curves) and R.G.B. scattering curves (broken curves) under $V_{\mathrm{V}}$ polarization for the specimen $\# 1$ (a) and \#2 (b). The structure parameters for each curve are given in Table II. 
angular region between 5 and 10 degrees. The difficulty arising from the interference effect in the assessment of the craze size and its refractive indices may be circumvented by studying the scattering at larger scattering angles, over much wider angular region, including the backward scattering. Studies of this kind may be important to further confirm the structure parameters evaluated in this work.

Finally we should like to point out some differences in the scattering behaviors predicted from the Mie theory and the R.G.B. theory. Figure 22 shows comparisons between the calculated Mie (solid curves) and R.G.B. scattering (broken curves) under the $V_{\mathrm{V}}$ condition for given sets of the structure parameters as given in the Table II: (a) for the specimen \#1 and (b) for the specimen \#2. It should be noted that the scattering curves obtained with the R.G.B. approximation show considerably large deviations from those obtained with the rigorous theory (Mie scattering). Thus the analysis based on the R.G.B. scattering can, in certain cases, cause significant errors in the assessment of the structure parameters.

\section{REFERENCES}

1. See for example, (a) R. P. Kambour, J. Polym. Sci., D, 7, 1 (1973); (b) S. Rabinowitz and P. Beardmore, C.R.C. Crit. Rev. Macromol. Sci., 1, 1 (1972).

2. R. P. Kambour and A. S. Holik, J. Polym. Sci., A-2, 7, 1393 (1969).
3. R. P. Kambour and R. R. Russell, Polymer, 12, 237 (1973).

4. P. Beahan, M. Bevis, and D. Hull, Philos. Mag., 24, 1267 (1971).

5. P. Beahan, M. Bevis, and D. Hull, J. Mater. Sci., A-2, 7, 183 (1969).

6. R. P. Kambour, Polymer, 5, 143 (1964).

7. R. P. Kambour, J. Polym. Sci., A, 2, 4159 (1964).

8. R. P. Kambour, J. Polym. Sci., A-2, 4, 349 (1966).

9. D. G. LeGrand, R. P. Kambour, and W. R. Haaf, $J$. Polym. Sci., A-2, 10, 1565 (1972).

10. E. J. Kramer, Bulletin of the American Physical Society, March, 1977, private communication.

11. P. Debye and A. M. Bueche, J. Appl. Phys., 20, 518 (1949).

12. P. Debye, H. R. Anderson, Jr., and H. Brumberger, $J$. Appl. Phys., 28, 679 (1957).

13. H. C. Van de Hulst, "Light Scattering by Small Particles," John Wiley \& Sons, Inc., New York, N.Y., 1957.

14. M. Kerker, "Scattering of Light and Other Electromagnetic Radiation," Academic Press, New York, N.Y., 1969.

15. J. A. Nelder and D. Mead, Comput. J., 7, 308 (1965).

16. Y. Uemura, M. Fujimura, T. Hashimoto, and H. Kawai, Polym. J., 10, 341 (1978).

17. Y. Uemura, T. Hashimoto, and H. Kawai, Sen-i Gakkaishi, 34, T-481 (1978).

18. Y. Uemura, T. Hashimoto, and H. Kawai, Polym. J., 11, 413 (1979).

19. A. Guinier and G. Fournet, "Small-Angle Scattering of X-rays," J. Wiley \& Sons., Inc., New York, N.Y., 1955, §2.1.5.

20. R.D. Andrews and T. J. Hammack, J. Polym. Sci., C, 5, 101 (1964). 